\title{
\begin{tabular}{l|l} 
Mibraries & DSpace@MIT
\end{tabular}
}

\author{
MIT Open Access Articles
}

Toward a closed loop from seismic imaging to earth-model building

The MIT Faculty has made this article openly available. Please share how this access benefits you. Your story matters.

Citation: Li, Yunyue Elita, Biondo Biondi, Dave Nichols, and Robert Clapp. “Toward a Closed Loop from Seismic Imaging to Earth-Model Building." The Leading Edge 35, no. 2 (February 2016): 135-139.

As Published: http://library.seg.org/doi/pdf/10.1190/tle35020135.1

Publisher: Society of Exploration Geophysicists

Persistent URL: http://hdl.handle.net/1721.1/103583

Version: Final published version: final published article, as it appeared in a journal, conference proceedings, or other formally published context

Terms of Use: Article is made available in accordance with the publisher's policy and may be subject to US copyright law. Please refer to the publisher's site for terms of use. 


\section{Toward a closed loop from seismic imaging to earth-model building}

Yunyue Elita $\mathrm{Li}^{1}$, Biondo Biondi ${ }^{2}$, Dave Nichols ${ }^{3}$, and Robert Clapp ${ }^{2}$

\begin{abstract}
Velocity-model building is the first task of seismic inversion and the foundation of the subsequent data-processing workflow. When the earth velocity becomes multivalued with respect to the propagating direction of the waves, velocity-model building becomes severely underdetermined and nonunique. The traditional workflow separates velocity-model building from lithologic inversion, which hampers both processing steps. An integrated model-building scheme is demonstrated to simultaneously consider prestack seismic data and its structural and lithologic inversion results from a previous iteration. The prestack seismic inversion is performed using wave-equation migration velocity analysis (WEMVA) for vertical transverse isotropic (VTI) models. To constrain the seismic inversion, the geologic information is integrated as spatial-model correlations, and the rock-physics information as lithologic-model correlations. This feedback step completes the loop from seismic imaging to lithologic-model building, where previous rock-physics estimations and geologic interpretations can be validated further and updated in order to constrain the next WEMVA iteration. Improvements from the integrated inversion scheme are shown on a Gulf of Mexico field data set.
\end{abstract}

\section{Introduction}

Anisotropic velocity-model building tries to resolve more than one parameter at each model location. This number could be three for a vertical transverse isotropic (VTI) media, increasing to five for a tilted transverse isotropic (TTI) media. For timerelated processing, seismic moveout in a VTI medium can be explained fully by two parameters: normal moveout (NMO) velocity $V_{\text {NMO }}$ and the anellipticity parameter $\eta$ (Tsvankin and Thomsen, 1994). In more recent developments of depth-processing workflows, advanced imaging and inversion techniques require anisotropic models that also can describe the anisotropic properties accurately within depth intervals. Parameterization studies of anisotropic waveform inversion show that ambiguity among the anisotropic parameters cannot be fully resolved without further constraining the parameters, e.g., by specifying functional relationships among them. Studies in 3D show that it is difficult to resolve a reliable and unique anisotropic model even with borehole-aided localized tomography.

To better constrain the anisotropic model building in this study, we include more knowledge of the subsurface in our seismic inversion, some of which knowledge might come from previous structural and lithologic inversion results. The structural knowledge is included in the form of spatial covariance of the parameters describing how smoothly the parameters vary in space. The lithologic knowledge is included as crossparameter covariance describing how one parameter varies with the other parameters at the same model location.

Here, our integrated inversion scheme inverts seismic data for VTI models using wave-equation migration velocity analysis (WEMVA) (Li and Biondi, 2011). The geologic and lithologic information, from a previous iteration of seismic processing, is feedback to the current inversion via gradient preconditioning. This method can be extended further for TTI and higher symmetries.

\section{Wave-equation migration velocity analysis for VTI models}

Anisotropic WEMVA aims at building an anisotropic earth model that minimizes the residual image from the surface seismic data. The image-space objective function can be written as:

$$
S(m)=\frac{1}{2}\left\|D_{\theta} I(x, \theta)\right\|_{2}^{2}-\frac{\alpha}{2}\left\|\sum_{\theta} I(x, \theta)\right\|_{2}^{2}+\frac{\beta}{2}\left\|c_{m}^{-\frac{1}{2}}\left(m-m_{p}\right)\right\|_{2}^{2},
$$

where the first two terms define the "data-fitting" objective, and the third defines the "model-regularization" objective. Model $m=(\mathrm{v}, \varepsilon, \sigma)$ is the VTI velocity model; $I(x, \theta)$ is the migrated image in the angle domain with $\theta$ the aperture angle and $\mathrm{D}_{\theta}$ a derivative operator along the angle axis. The first term is intended to minimize the differential semblance in the angle gathers, and the second term is intended to maximize the stack power. In the third objective, $m_{p}$ and $\mathrm{C}_{m}$ define a Gaussian distribution of a prior model that is ideally independent of the seismic data. This regularization will bring more information into the optimization and stabilize the inversion. Parameters $\alpha$ and $\beta$ balance the relative weights among different objectives.

The data-fitting objectives tend to be more sensitive to velocity than to anisotropic parameters, especially at near and intermediate angles. Therefore, the objective function is biased toward the velocity error despite the error in the other VTI parameters. When the velocity is inaccurate, the gradient directions of the data-fitting objectives could be overwhelmed by the velocity error, possibly misguiding updates for the anisotropic parameters.

\section{Geologic and rock-physics constraints}

Assuming a Gaussian distribution, Tarantola (1984) includes the prior information using the mean and the covariance of the model as a regularization term. We separate the covariance into two parts: (1) a spatial covariance between the same parameter at different locations; and (2) a crossparameter covariance between different parameters at the same location. The spatial covariance is defined mainly by the geologic structure in the neighborhood

${ }^{1}$ Formerly Stanford University; now Massachusetts Institute of Technology.

${ }^{2}$ Stanford University.

${ }^{3}$ Schlumberger. 
of a given imaging location, and it can be estimated using a set of smoothing filters from the migrated image. The crossparameter covariance can be inferred from the lithologic information at the model location.

We focus on shale-induced anisotropy and assume that spatial covariance and local crossparameter covariance components are mutually independent. To speed up convergence of the iterative inversion, we use a preconditioning scheme. Mathematically, we express the preconditioning variable $n$ to the original anisotropic model $m$ by:

$$
m=\Sigma \mathrm{S} n
$$

where the smoothing operator $\mathrm{S}$ is a band-limited diagonal matrix with potentially different smoothing operators for velocity, $\varepsilon$, and $\delta$, according to the geologic information in the study area. The standard deviation matrix $\Sigma$ is the square root of the crossparameter covariance matrix. We illustrate the procedure to obtain this additional information from stochastic rock-physics modeling using the following field example. a

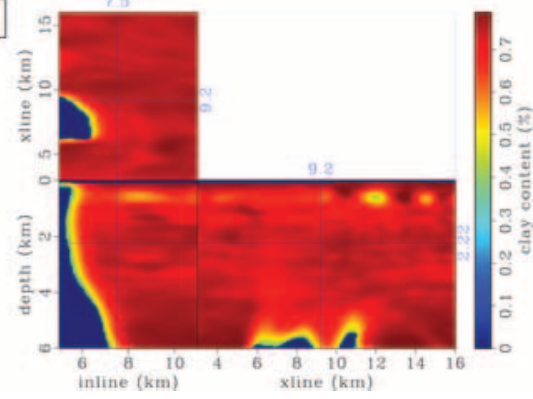

b

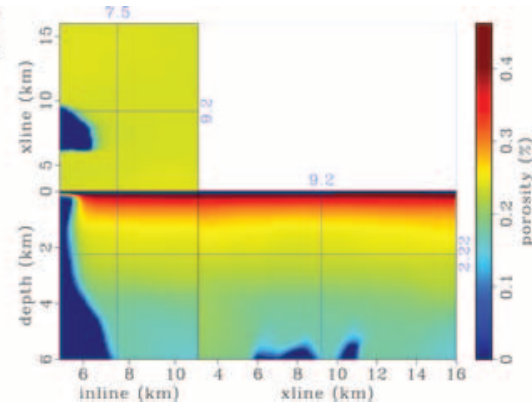

Figure 1. Lithologic inversion results from initial data processing in (a) inverted clay content and in (b) inverted porosity. a

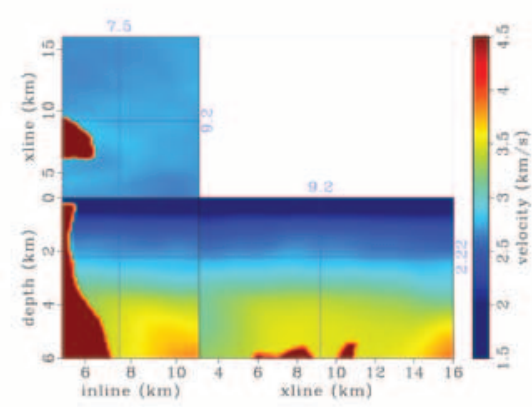

C

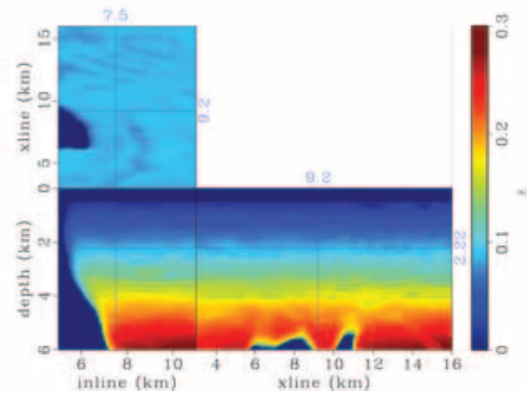

b
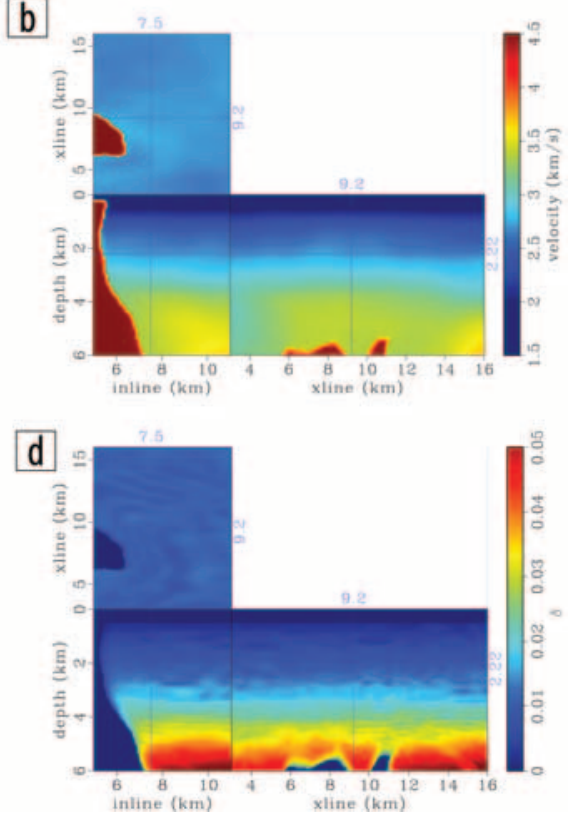

Figure 2. Isotropic tomography velocity model is shown in (a). Anisotropic parameters $\mathrm{v}_{0}, \varepsilon$, and $\delta$ are shown in (b), (c), and (d), respectively. Parameters $\varepsilon$ and $\delta$ are the mean models from the stochastic rock-physics modeling. Vertical velocity $\mathrm{v}_{0}$ is computed from the isotropic velocity and the $\delta$ model to honor the seismic traveltime.

\section{Gulf of Mexico field data example}

The data we use in this article were acquired in the Gulf of Mexico. The sedimentary basin has been filled by shaley sandstones. Therefore, we focus on shale anisotropy during seismic inversion and rock-physics-model building. We combine the rock-physics models proposed by Bachrach (2010) and Bandyopadhyay (2009) to consider the intrinsic mineral anisotropy, particle alignment, and clay mineral transition during compaction. By varying input parameters of the rock-physics model, we explore different shale-rock scenarios. These models are the sources of the prior rock-physics knowledge (Li et al., 2014).

Schlumberger MultiClient performed an initial ray-based tomography on this data set, producing isotropic velocity along with lithologic inversion results, such as shale content and porosity. Therefore, we use the provided shale content inversion cube (Figure 1a) and a smoothly varying porosity trend (Figure $1 \mathrm{~b}$ ) as the input to the rock-physics model. To include the spatial uncertainty of the lithologic inversion result, the statistics of each estimated parameter are evaluated within a window around each model location, allowing the lithologic estimation to include variations within a few seismic wavelengths.

Figure 2a shows the provided isotropic velocity model from the initial tomography. Figures $2 \mathrm{~b}$ to $2 \mathrm{~d}$ show the initial anisotropic model. The initial $\varepsilon$ model (Figure $2 \mathrm{c}$ ) and $\delta$ model (Figure $2 \mathrm{~d}$ ) are the mean models from the stochastic rockphysics modeling. The salt body and the water column are considered isotropic. Figure 3 compares the images obtained from isotropic migration and anisotropic migration. The anisotropic migration using models in Figure 2 improves the focusing of the events highlighted by the labels. However, the overall imaging quality of both migrated images is similar. These images demonstrate a typical case where two different models, with completely different geologic implications, can explain the seismic kinematics equally well. These structural images are used to estimate the steering filters to precondition the spatial distribution of the parameters.

More importantly than producing a single model, the stochastic rock-physics modeling also allows us to explore possible ranges of the anisotropic parameters. At a particular model location, we obtain an ensemble of $\mathrm{v}_{0}, \varepsilon$, and $\delta$ values as shown in Figure 4. Assuming the three parameters $\mathrm{v}_{0}, \varepsilon$, and $\delta$ follow a multivariate Gaussian distribution, we summarize their variance using a $3 \times 3$ matrix with six independent elements. This crosscovariance matrix is estimated at each subsurface location. 
Figures $5 \mathrm{a}$ to $5 \mathrm{c}$ show the diagonal elements in the square root of the covariance matrix. The strong lateral variations in vertical velocity variance (Figure $5 \mathrm{a}$ ) and in $\varepsilon$ variance (Figure $5 b)$ show that their uncertainties are strongly correlated with the lithology. The $\delta$ variance (Figure 5c) shows less lateral variation, indicating that parameter $\delta$ is controlled mainly by compaction and mineral transition. Figures $5 \mathrm{~d}$ to $5 \mathrm{f}$ show the off-diagonal elements in the standard deviation matrix. The crosscovariances between $\mathrm{v}_{0}$ and $\varepsilon$ (Figure $5 \mathrm{~d}$ ) as well as between $\mathrm{v}_{0}$ and $\delta$ (Figure 5 e) suggest that velocity and anisotropy are negatively correlated in the shallow region but positively correlated in the deep region. In the shallow region, high velocity correlates with low anisotropic sand; whereas in the deeper region, high velocity can be caused by mineral diagenesis from smectite to illite, which is also highly anisotropic (Hornby et al., 1995). Covariance between $\varepsilon$ and $\delta$ (Figure $5 f$ ) shows positive correlations for all depths.

The gradients of the WEMVA objective function in the first iteration with respect to model parameters are shown in Figure 6. Compared with the gradient in $\mathrm{v}_{0}$ (Figure 6a), the gradients in $\varepsilon$ (Figure 6b) and $\delta$ (Figure 6c) show similar structure and point to the same update directions. These updates are determined by the WEMVA tomographic operator in order to increase the stack power. However, the negative correlations between velocity and anisotropy in the shallow region suggest that velocity and anisotropy should vary in opposite directions. As a result, the gradient directions in $\varepsilon$ (Figure 6e) and $\delta$ (Figure 6f) in the shallow region have been reversed after the rock-physics preconditioning. These preconditioned gradients (Figure 6) are used in a Polak-Ribiére

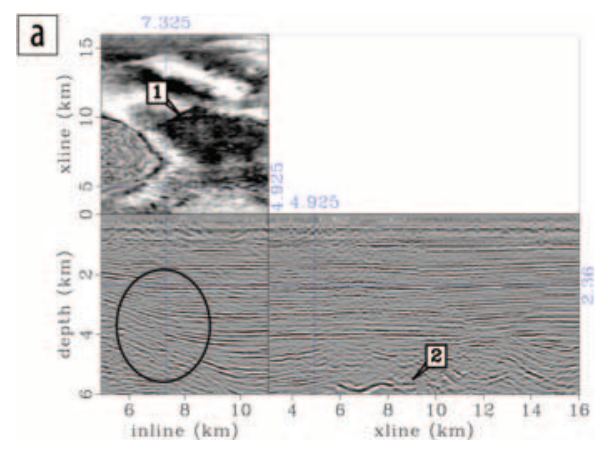

b

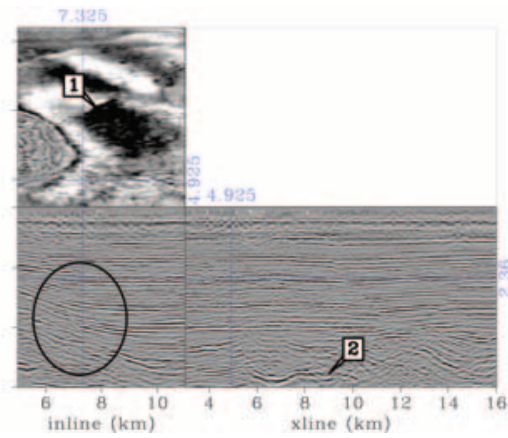

Figure 3. Images obtained from (a) isotropic migration and from (b) anisotropic migration with the models in Figure 2. The fault (label 1) on the depth slice appears to be better focused on the anisotropic migration image. The steeply dipping reflectors (circled on the in-line section) and the top of a salt segment (label 2) are more continuous on the anisotropic migration image. Nonetheless, the overall quality of both images is quite similar. These images illustrate a typical situation where different models can explain the seismic data equally well.
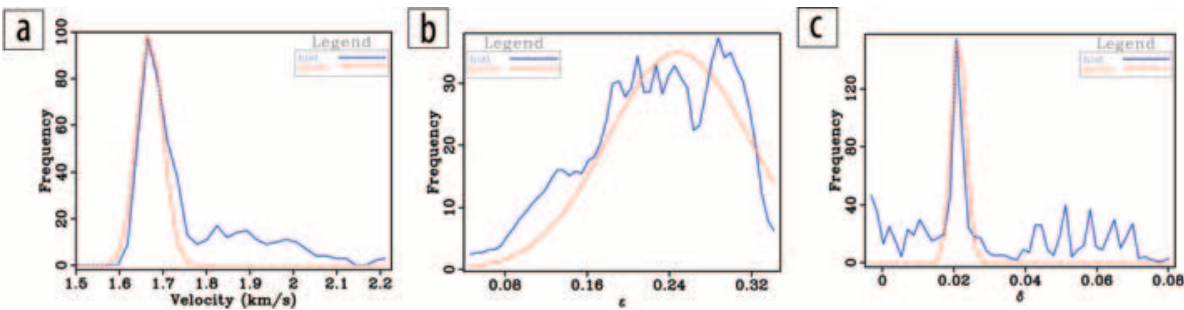

Figure 4. Histogram and its Gaussian approximation at a single location of (a) $\mathrm{v}_{0}$, (b) $\varepsilon$, and (c) $\delta$. The histograms are obtained from stochastic rock-physics modeling. Their multivariate Gaussian approximations are used in the preconditioned WEMVA inversion.

a

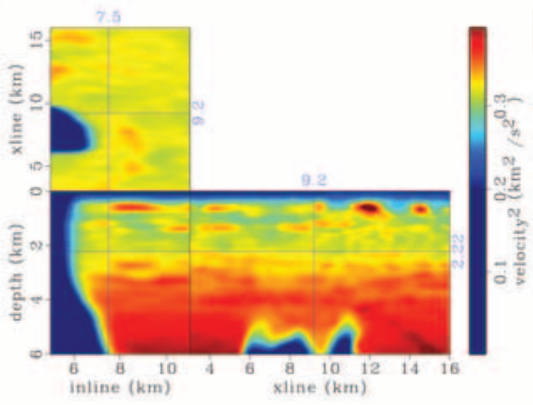

b

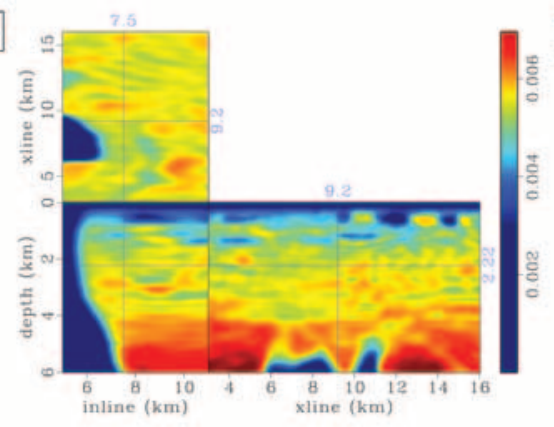

\section{C}

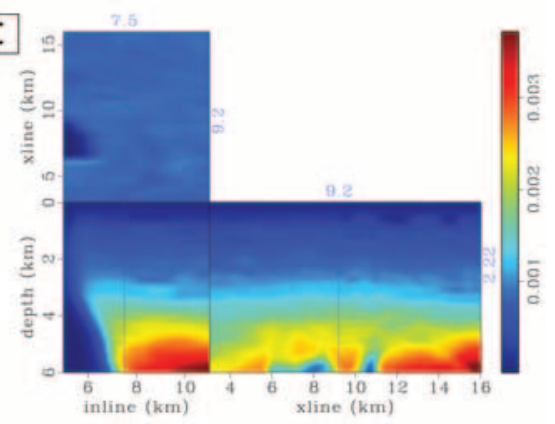

$f$

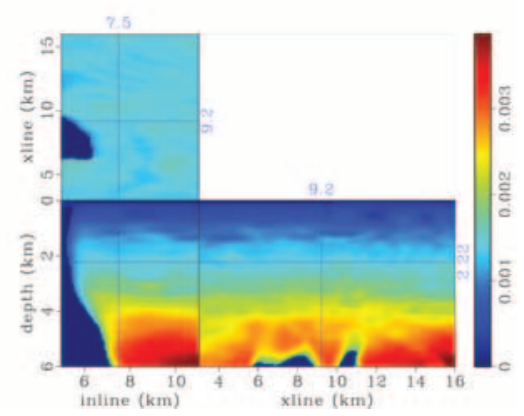

d

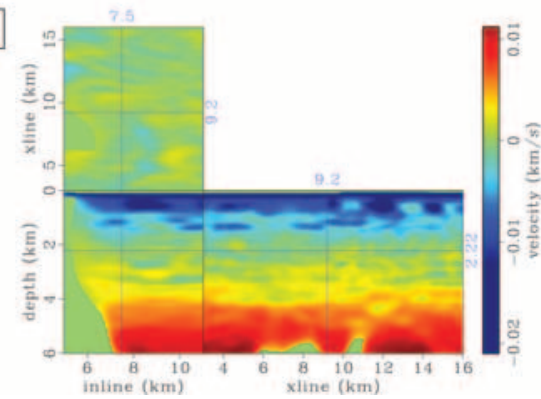

e

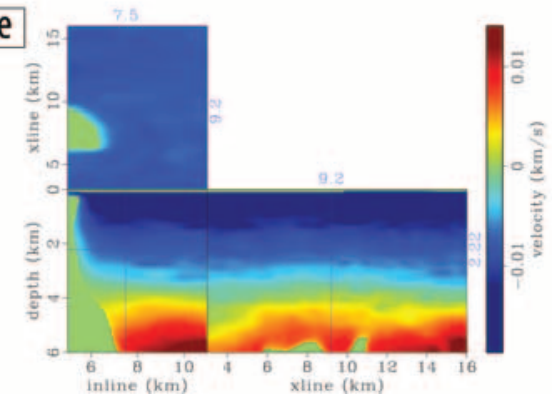

Figure 5. Six independent components of the crossparameter covariance maxtrix with (a) $C_{v v}$, (b) $C_{e e}$, (c) $C_{d d}$, (d) $C_{v e}$, (e) $C_{v d}$, and (f) $C_{e d}$. This matrix
is obtained from stochastic rock-physics modeling. It captures the crosscorrelations between the anisotropic parameters based on a sandy shale model. 
a

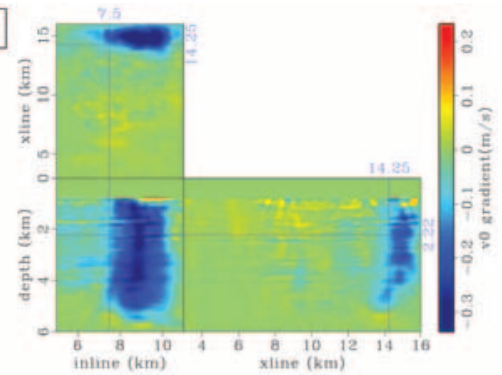

d

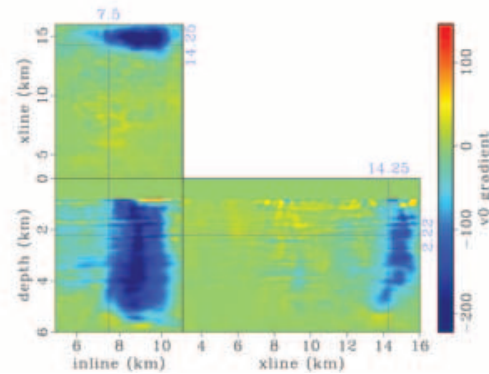

b

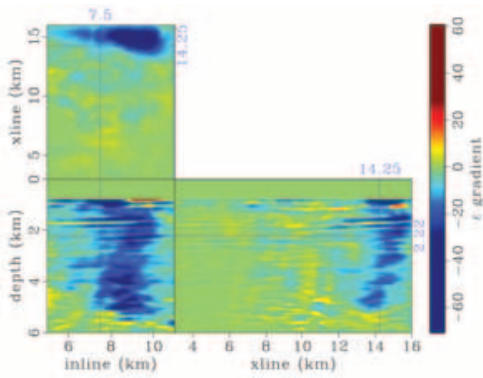

e

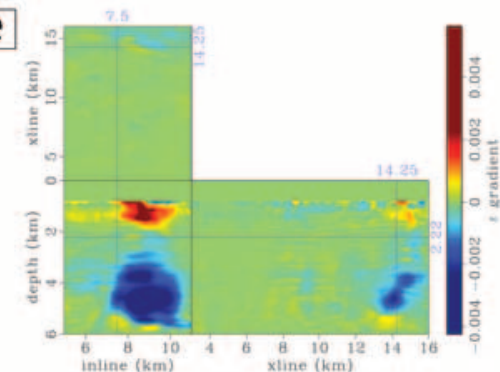

C

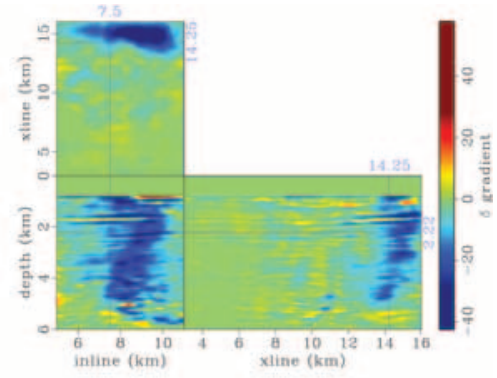

f

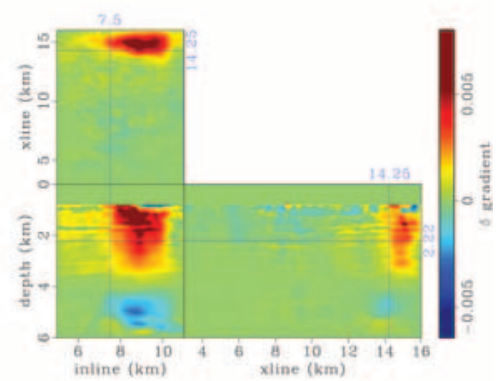

Figure 6. Gradients for the VTI parameters of the first WEMVA iteration before (a, b, c) and after (d, e, f) rock-physics preconditioning. From left to right, columns display gradients of (a and d) $\mathrm{v}_{0},(\mathrm{~b}$ and $\mathrm{e}) \varepsilon$, and ( $\mathrm{c}$ and $\left.\mathrm{f}\right) \delta$, respectively.

a

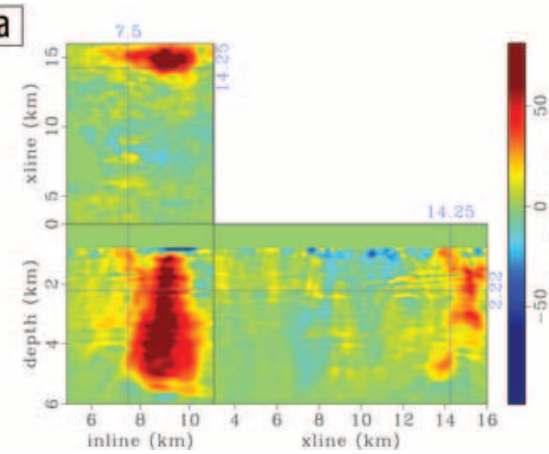

b

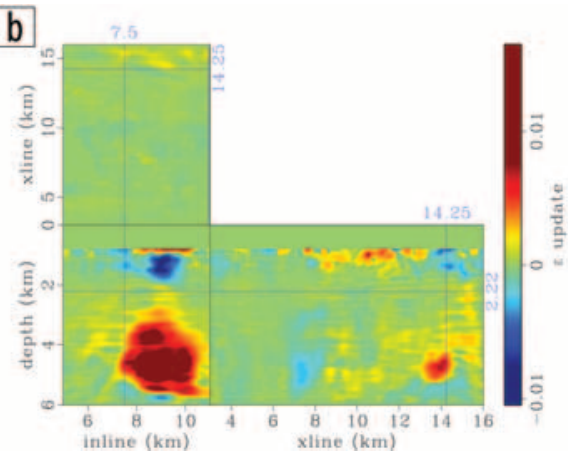

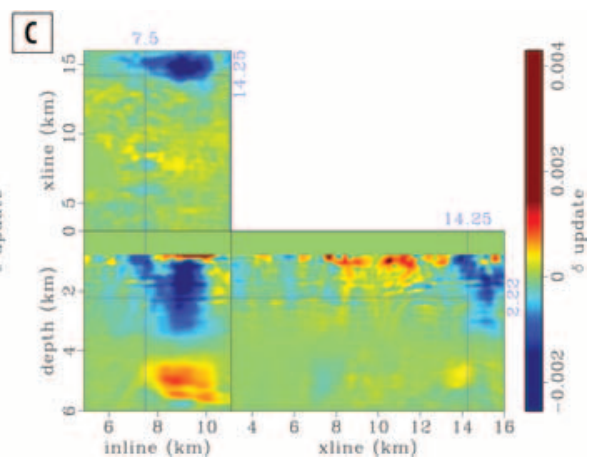

Figure 7. Total updates (a) in $\mathrm{v}_{0}$, (b) in $\varepsilon$, and (c) in $\delta$ after 10 nonlinear WEMVA iterations. The inversion picks up a high-velocity anomaly on the hanging fault.

nonlinear conjugate gradient scheme to calculate the update directions for each parameter.

Figure 7 shows the total updates in the VTI model after 10 nonlinear iterations. The inversion identifies a positive velocity anomaly on the hanging wall of the normal fault. To consistently produce a high-velocity rock in the shallow region, the regularization scheme reduces the anisotropy in the corresponding region, consistent with a stronger sand-dominated sedimentary environment. In the deeper region, both velocity and anisotropy are positively updated, consistent with the strong diagenesis process in shale. Significant updates also are shown around the salt body where the initial ray-based tomography failed to update. On average there are $5 \%$ positive updates in vertical velocity and more than $10 \%$ updates in both $\varepsilon$ and $\delta$.

Figure 8 compares migrated images using the initial model with the migrated images using the updated model at a crossline location near the salt flank. Reflectors in both inline and crossline directions are strongly dipping. Highlighted by circles labeled 2 and 3 , these reflectors are broken in the initial image, making the interpretation of the sediments around the salt body a challenging task. The updated model reveals a much clearer image around the salt. Reflectors in both areas are better imaged with more continuity and better resolution. On the depth slice, label 1 points to a fault that is blurred in the initial image but is distinctly imaged with the updated model.

In the upper-right corners of Figures $8 \mathrm{a}$ and $8 \mathrm{~b}$, we compare the angle-domain common image gathers (ADCIGs) before and after inversion. The initial ADCIGs are reasonably flat, with only minor residual moveout. Nevertheless, the flatness of the ADCIGs has been improved with the updated VTI model. Without changing depth dramatically, the ADCIGs show increased angle coverage in the shallow region, mostly due to the improved definition in the Thomsen parameters $\varepsilon$ and $\delta$.

\section{Discussion}

Earth-model building is a highly underdetermined problem, and uncertainties propagate from each step of the model-building process. In this study, we include the uncertainties of the shaleanisotropy modeling by sampling the distribution of the input parameters. However, there are more uncertainties with different 
a

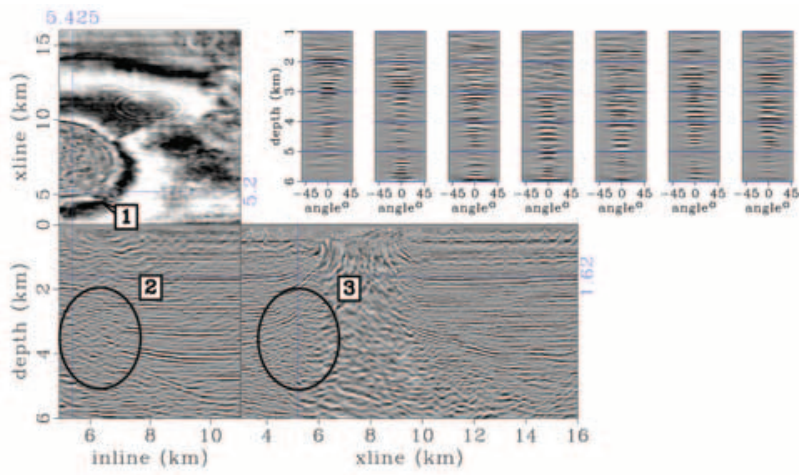

b

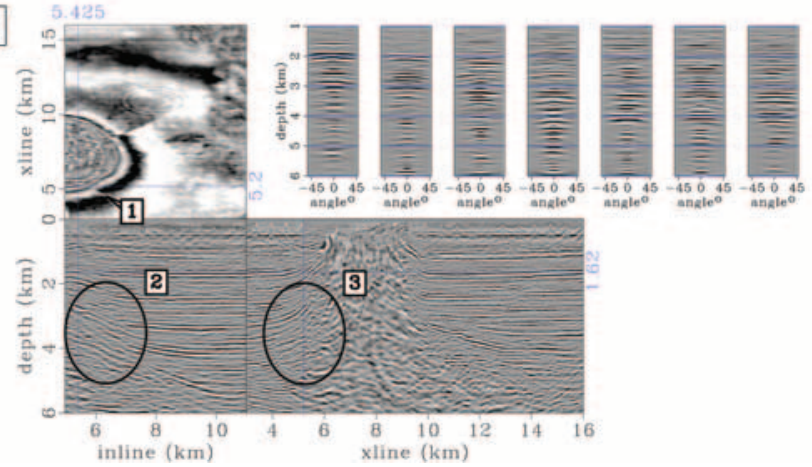

Figure 8. Comparison between the migration images near the salt body (a) using the initial anisotropic model and (b) using the inverted anisotropic model. The fault on the depth slice (label 1 ) is better focused on the updated image. Circles 2 and 3 point out two regions where the updated anisotropic model produces a better image with better continuity and higher resolution. Angle-domain common-image gathers are further flattened using the inverted anisotropic model.

geologic scenarios that have been neglected, such as stress-induced or fracture-induced anisotropy. Moreover, the seismic-data inversion for velocity and rock properties is also nonunique. The current inversion practice of producing a single solution lacks uncertainty quantification, which can be highly valuable for decision making and risk management.

Using previous seismic inversion results to constrain the seismic model building for the next model-building iteration helps us close the loop linking seismic data with reservoir modeling. Traditional processing from seismic data to a reservoir model does not include feedback; because of this, the seismic data modeled from the inverted reservoir model usually do not match the field data. This study provides a step toward building a closed loop from exploration to production. With more iterations, earth models that are consistent with all the available data can be more reliably estimated and their uncertainties evaluated.

\section{Conclusion}

We tested the rock-physics-constrained anisotropic WEMVA method on a 3D field data set. We first built the rock-physics constraints using stochastic rock-physics modeling and then utilized these constraints during anisotropic WEMVA inversion to produce reliable VTI models as well as better-focused images. A TTI migration would improve the image further, especially for the salt flank. However, it is out of the scope of this article, mainly due to the computational constraints.
Stochastic rock-physics modeling is a powerful tool to model shale anisotropy and to explore the possible ranges of the anisotropic parameters. By sampling the distributions of key parameters, we include their uncertainties and produce an ensemble of anisotropic models that are realizable by rock-physics modeling. The field data example demonstrates that it is reasonable to assume a multivariate Gaussian distribution and summarize the random variables using the mean and the covariance matrix. Migrated images based on the mean model demonstrate that the rock-physics modeling produces good initial anisotropic models for seismic imaging. A 3D prior distribution model is made possible by utilizing the lithologic inversion results from a previous seismic-processing workflow.

By constraining anisotropic WEMVA with the geologic and rock-physics covariance, we feed the prior rock-physics information back to the seismic data inversion, which significantly improves the convergence. The inverted VTI model not only explains the reflection data (flattens the gathers), but also follows basic geologic and rock-physics principles. The 3D example in this study demonstrates that anisotropic WEMVA can improve the subsurface model further and focus the subsurface image further, especially for the steeply dipping reflectors around the salt body. TIL:

\section{Acknowledgments}

The authors thank Sam Gray for the invitation of this article. We thank Schlumberger MultiClient for providing the field data set. We acknowledge the Stanford Center for Computational Earth and Environmental Science for providing computing resources. Yunyue Elita Li acknowledges the sponsors of the Stanford Exploration Project for their financial support of this study.

Corresponding author: yunyue.li@sep.stanford.edu

\section{References}

Bachrach, R., 2010, Applications of deterministic and stochastic rock physics modeling to anisotropic velocity model building: 80th Annual International Meeting, SEG, Expanded Abstracts, 2436-2440, http://dx.doi.org/10.1190/1.3513342.

Bandyopadhyay, K., 2009, Seismic anisotropy: Geological causes and its implications: Ph.D. thesis, Stanford University.

Hornby, B., D. Miller, C. Esmersoy, and P. Christie, 1995, Ultrasonicto-seismic measurements of shale anisotropy in a North Sea well: 65th Annual International Meeting, SEG, Expanded Abstracts, 17-21, http://dx.doi.org/10.1190/1.1887505.

Li, Y., and B. Biondi, 2011, Migration velocity analysis for anisotropic models: 81st Annual International Meeting, SEG, Expanded Abstracts, 201-206, http://dx.doi.org/10.1190/1.3627605.

Li, Y., G. Mavko, and D. Nichols, 2014, Stochastic rock physics modeling for seismic anisotropy: 84th Annual International Meeting, SEG, Expanded Abstracts, 2899-2904, http://dx.doi. org/10.1190/segam2014-0299.1.

Tarantola, A., 1984, Inversion of seismic reflection data in the acoustic approximation: Geophysics, 49, no. 8, 1259-1266, http://dx.doi. org/10.1190/1.1441754.

Tsvankin, I., and L. Thomsen, 1994, Nonhyperbolic reflection moveout in anisotropic media: Geophysics, 59, no. 8, 12901304, http://dx.doi.org/10.1190/1.1443686. 